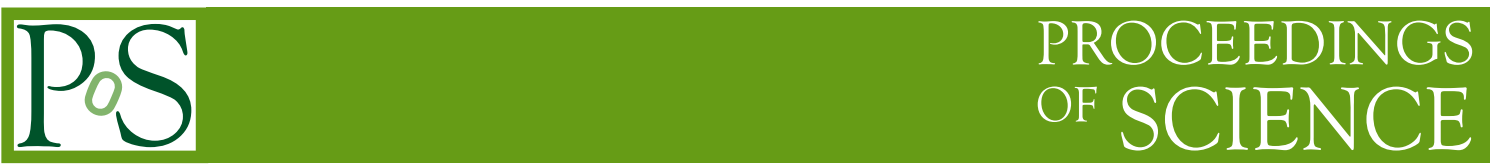

\title{
Review of Dark Matter searches with Cherenkov telescopes
}

\author{
Javier Rico* \\ Institut de Física d'Altes Energies, Barcelona, Spain \\ E-mail: jricoeifae.es
}

Dark matter distributes in the Universe in halos that harbor galaxy clusters, galaxies of different sizes, or even dark matter "clumps" which have not attracted enough mass to start stellar activity. Gamma-ray instruments search for dark matter by looking for gamma rays coming from these sites, and comparing the results of their observations with the expectations for different dark matter models. Cherenkov telescopes, sensitive to gamma rays in the energy range between $\sim 100$ $\mathrm{GeV}$ and $\sim 10 \mathrm{TeV}$, are suited for searches of dark matter with mass above several $100 \mathrm{GeV}$. In this paper, I review the results obtained by the current generation of Cherenkov telescopes (MAGIC, VERITAS and H.E.S.S.) in this kind of searches.

Science with the New Generation of High Energy Gamma-ray experiments, 10th Workshop 04-06 June 2014

Lisbon - Portugal

* Speaker. 


\section{Introduction}

There is overwhelming experimental evidence for the existence of dark matter, mainly from its gravitational effects on the dynamics of galaxies and galaxy clusters and measurements of the power spectrum of temperature anisotropies of the Cosmic Microwave Background [1]. The latter shows that dark matter accounts for about $85 \%$ and $27 \%$, respectively, of the total mass and energy contents of the Universe.

Our current cosmological model is based on the Cold Dark Matter paradigm, where dark matter is composed of electrically neutral, weakly interacting particles that are stable within cosmological time scales. These particles also have to be cold -i.e., non-relativistic at the onset of large-structure formation-, in order to produce the relic density required by the cosmological observations and to be compatible with the Big Bang nucleosynthesis. Such particles do not exist within the Standard Model (SM) of Particle Physics, so one must go beyond its borders in search for the suitable dark matter candidates.

A particularly well-motivated class are the weakly interacting massive particles (WIMPs, [2]). WIMPs have a mass in the range between $10 \mathrm{GeV}$ and a few $\mathrm{TeV}$, and interaction cross-sections typical of the weak scale. They naturally produce the needed relic density and annihilate or decay into SM particles detectable by the current or near future generation of experiments. WIMP searches are based on three different experimental strategies: production at accelerators such as the Large Hadron collider [3], direct detection from measurements of the nuclear recoils from WIMP scattering off standard particles in underground detectors [4], and indirect detection through the identification of SM particles coming from WIMP annihilation or decay processes in astronomical dark matter over-density sites. These approaches are complementary and explore independent regions of the parameter space [5]; hence each strategy has to be pursued in order to form a global perspective of dark matter searches.

The case of WIMP indirect detection via gamma rays and charged particles (electrons and positrons) is of particular interest, and the main subject of this paper. There are other plausible cold dark matter candidates (see, e.g., [6]), including some, e.g. axion-like particles, that could produce experimental signatures in the gamma-ray domain [7, 8, 9], which we will not cover in detail in this paper.

\subsection{Indirect dark matter searches with gamma rays}

WIMPs can be searched for indirectly through the identification of gamma rays from annihilation or decay processes in dark matter over-density sites. The expected gamma-ray flux from a region of the sky $\Omega$ can be factorized as $d \phi / d E=d \phi^{\mathrm{PP}} / d E \times J(\Omega)$, where:

$$
\begin{aligned}
& \frac{d \phi^{\mathrm{PP}}}{d E}=A \frac{d N_{\gamma}}{d E} \\
& J(\Omega)=\frac{1}{4 \pi} \int_{\Omega} \int_{\mathrm{los}} \rho^{k}(l) d l d \Omega
\end{aligned}
$$

In the particle physics term, $d \phi^{\mathrm{PP}} / d E, A=\langle\sigma v\rangle / 2 m_{\mathrm{dm}}^{2}$ for annihilation or $1 / m_{\mathrm{dm}} \tau_{\mathrm{dm}}$ for decay; $\langle\sigma v\rangle$ is the velocity-averaged annihilation cross section of the dark matter particle; $m_{\mathrm{dm}}$ and $\tau_{\mathrm{dm}}$ are the mass and lifetime of the dark matter particle, respectively, $N_{\gamma}$ is the number of gamma 
rays produced per annihilation or decay reaction, and $E$ the energy of the gamma ray. In the astrophysical term $J(\Omega)$ (often referred to as J-factor), $\rho$ is the dark matter density, with $k=2$ for annihilation and $k=1$ for decay. The integrals run over the line of sight $(l)$ and the observed sky region $(\Omega)$.

$d \phi^{\mathrm{PP}} / d E$ contains all the information dependent on the specific nature of the dark matter particle and its interactions, i.e., it is completely determined for a given theoretical framework. $d N_{\gamma} / d E$ contains the spectral information. Using the a priori knowledge of its shape is the key for an optimal dark matter-oriented analysis strategy [10]. Gamma rays can be produced as primary, radiative, or secondary products in annihilation or decay reactions. Reactions yielding primary gamma rays include annihilations or decays into two gamma rays or a gamma ray and another electrically neutral particle, which result in monochromatic lines in the spectrum. Gamma rays may also be produced in radiative processes, either by final state or virtual charged particles [11]. This contribution normally dominates the spectrum close to the kinematical limit at $\sim m_{\mathrm{dm}}$ and, more importantly, produces characteristic spectral features that can be used to distinguish a possible dark matter signal from astrophysical background. A third contribution, with less characteristic spectral features, comes from secondary processes, i.e. hadronization and decay of the products of the dark matter decay or annihilation [12]. In addition, gamma rays can result from the inverse Compton up-scattering of photons in radiation fields by primary or secondary charged particles. For energies of the order of the dark matter particle mass, this component is subdominant with respect to the previous ones, and will be henceforth neglected.

$J(\Omega)$ determines the signal intensity and contains all the information regarding the source distance and geometry. Dark matter forms halos in a hierarchical fashion, from dwarf galactic satellites to clusters of galaxies, through collision-less gravitational collapse. The characteristics of dark matter halos are computed by fitting the results of N-body simulations to the measurements of velocities of the luminous matter. The simulations lead to similar final equilibrium state for any mass range, from micro-haloes to galaxy cluster, over a mass range spanning 20 decades independent of the small-scale structure and hierarchical merging pattern [13, 14].

\subsection{Observational targets}

Most aspects of the general cold dark matter halo structure resulting from gravitational collapse are well resolved, and different numerical approaches basically agree. Our detailed understanding and predictive power is now limited by the complex and poorly understood interplay between the dark matter and baryonic components (for a detailed discussion see, e.g. [6]). In addition, while the success of the Cold Dark Matter paradigm is due to the good agreement of its predictions with observations for the large-scale structures, the N-body simulations appear to disagree with the astronomical observations on small scales, which might indicate that the dark matter properties differ from the standard paradigm. In particular, if the DM particles could self-interact, one could get a better model-data agreement for central densities of dwarf spheroidal galaxies and galaxy clusters [15, 16], and obtain an experimental signature in the gamma-ray domain [17].

Hierarchical dark matter merging gives rise to a vast amount of substructure, providing us with suitable targets for indirect dark matter searches at different scales, namely:

- Clusters of galaxies. They are the largest structures known in our Universe, with typically 
up to $80 \%$ of their total mass in the form of non-baryonic dark matter, which makes them good sites for indirect dark matter searches despite the fact that they are located at very large distances. Additionally, substructure could significantly enhance the annihilation signal above that predicted for a smooth halo. However, we expect intense gamma-ray background signals from cosmic ray interactions or AGNs in the cluster [18].

- Galactic center and halo. The most intense dark matter-induced gamma-ray signals are expected to come from the Galactic center. The intense gamma-ray background produced by the central source and cosmic ray interactions with interstellar matter can be significantly reduced by observing the halo slightly offset the center, which still provides the highest expected J-factor among all known possible sources. The main drawback comes from the large uncertainties on the dark matter distribution below few hundred parsec, which affect this kind of searches [19].

- Mini-halos and satellite galaxies. N-body simulations predict the existence of pure dark matter sub-haloes within our Galaxy. Some of them have attracted enough barionic mass to start stellar activity and form satellite galaxies, whereas other remain fully dark. Satellite galaxies have very high mass-to-light ratios (they are the most dark matter-dominated systems known so far [20]). These kind of targets have the advantage of being free of astrophysical gamma ray sources and, because they are relatively close, they appear as (quasi-)point-like sources for Cherenkov telescopes, with relatively high J-factors, albeit typically below the one expected for the Galactic Center.

\subsection{Indirect dark matter searches with Cherenkov telescopes}

WIMP annihilation and decay produce gamma rays in the GeV-TeV energy range, which are searched for by gamma-ray instruments observing the targets described in the previous section. Currently, that energy window is best explored from space, by the Large Area Telescope (LAT) onboard the Fermi satellite, and from ground, by the Cherenkov telescopes MAGIC, VERITAS and H.E.S.S. Cherenkov telescopes are sensitive to gammas of very high energies, between few tens of $\mathrm{GeV}$ and $\sim 100 \mathrm{TeV}$. With a typical field of view of few (3-5) degrees in diameter, they are optimized to operate in pointing mode. Since they operate under (quasi-) dark conditions, their duty cycle is $\sim 10-15 \%$. All this results in a high level of competition for the observation time between different proposed targets and physics objectives. Although the primary goals of Cherenkov observatories deal with high-energy astrophysics, their scientific programs also include questions of Fundamental Physics [21], and particularly dark matter searches, which so far have not produced any positive result. In this paper we review the most relevant results produced by Cherenkov telescopes in the search for dark matter signals.

\section{Experimental results on dark matter searches by Cherenkov telescopes}

\subsection{Results from the Galactic center and halo}

One of the first results obtained by the present generation of Cherenkov telescopes is that of the H.E.S.S. observations of the Galactic center during 2003 (17 hours, from $200 \mathrm{GeV}$ to $10 \mathrm{TeV}$ ) 

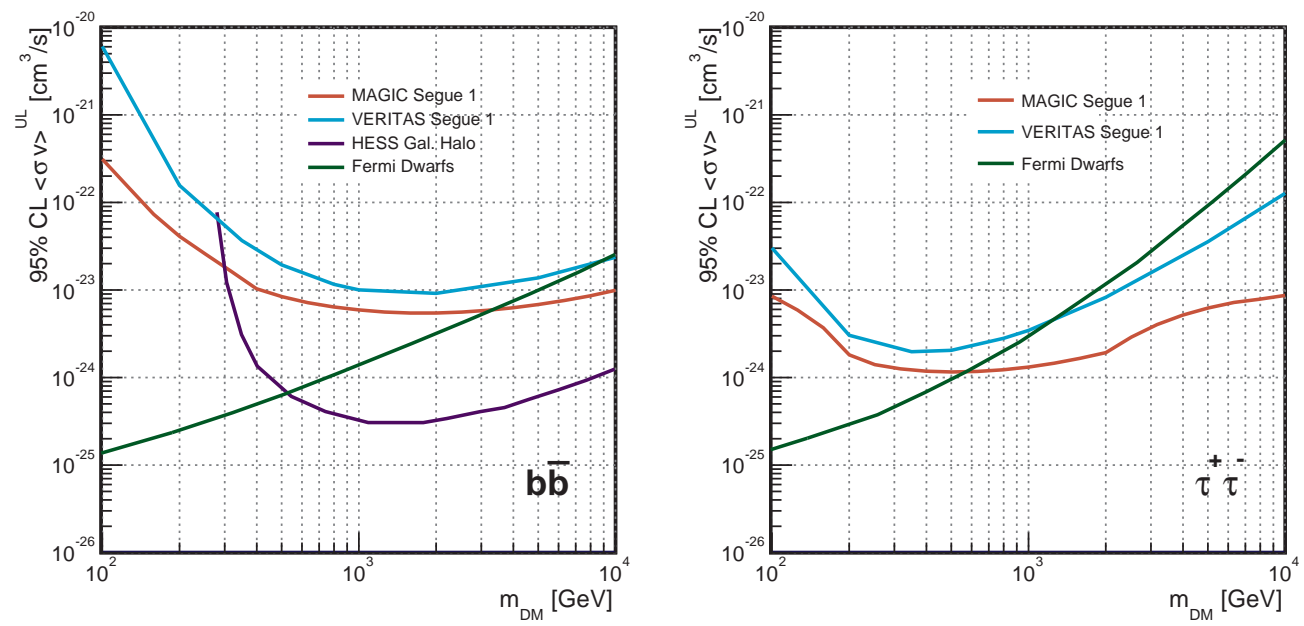

Figure 1: Comparison of results from indirect dark matter searches with gamma rays, including the most relevant results by Cherenkov telescopes (MAGIC observations of Segue 1 [25], VERITAS observations of Segue 1 [26] and H.E.S.S. observations of the Galactic center region[19]) compared to the results by FermiLAT on dwarf spheroidals [27]. On the left (right), a decay into $b \bar{b}\left(\tau^{+} \tau^{-}\right)$quarks (leptons) is assumed. Figure adapted from [25].

and 2004 (49 hours, from $200 \mathrm{GeV}$ to $30 \mathrm{TeV}$ ) [22]. The results confirmed the presence of a central, steady, point-like source, with a spectrum well described by a power law up to several tens of TeV, and with no indication of any dark matter characteristic spectral feature. The detected signal was therefore concluded to most likely be of non-dark matter origin, and limits to $\langle\sigma v\rangle$ of the order of $10^{-24}-10^{-23} \mathrm{~cm}^{3} \mathrm{~s}^{-1}$ could be inferred. Later observations by MAGIC [23] and VERITAS [24] are in agreement with these conclusions.

The presence of a strong astrophysical background close to the Galactic center can be overcome by observing nearby regions, where its contribution is relatively low, whereas the expected dark matter contribution is still significant. H.E.S.S. has performed this kind of search between 2004 and 2008 [19]. They accumulated 112 hours of observations of a region of radius $1^{\circ}$ centered at the Galactic center, restricting the search for dark matter to the region of Galactic latitude $|b|>0.3^{\circ}$. The projected distance of the considered region corresponds to $45-150 \mathrm{pc}$ from the Galactic center. This approach has the advantage that several popular parameterizations of the dark matter distribution in the Galaxy yield similar predictions for that region, albeit is not valid for an isothermal distribution. The results of this analysis are the most constringent for masses above $\sim 500 \mathrm{GeV}$, excluding values of the velocity-averaged annihilation cross-section down to $\langle\sigma v\rangle \sim 3 \times 10^{-25} \mathrm{~cm}^{3} \mathrm{~s}^{-1}$ for $\sim 1 \mathrm{TeV}$ particles annihilating into $b \bar{b}$ quark pairs (see Figure 1).

H.E.S.S. has also used their observations of the Galactic center region to look for gammaray line signatures [28]. The detection of such a spectral feature would constitute a smoking-gun for dark matter, since no known astrophysical process can produce such a signature at the $\mathrm{GeV}$ $\mathrm{TeV}$ energies. H.E.S.S. has set the most constraining upper limits in the mass range between $\sim$ $500 \mathrm{GeV}$ and $\sim 25 \mathrm{TeV}$. For a dark matter particle mass of $1 \mathrm{TeV}$, the limits to the velocity averaged annihilation cross section into two gamma rays is $\langle\sigma v\rangle=3 \times 10^{-27} \mathrm{~cm}^{3} \mathrm{~s}^{-1}$ (see Figure 2). 


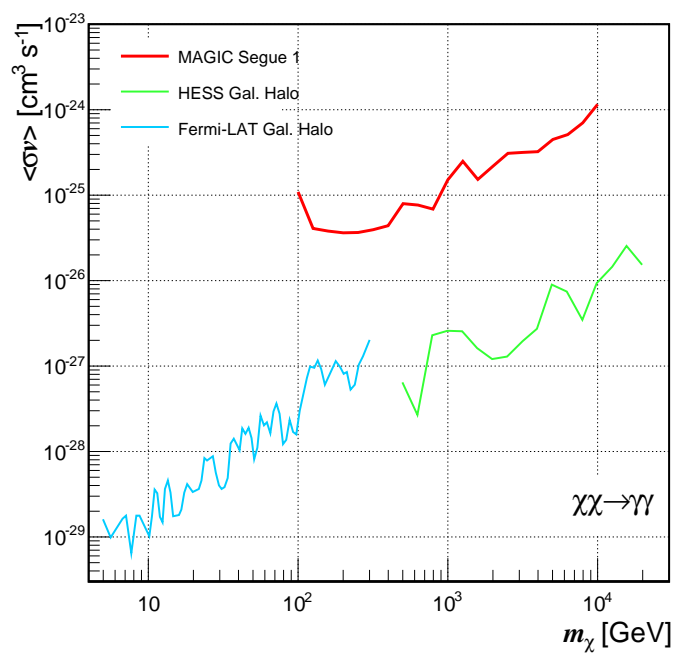

Figure 2: Comparison of results of searches for gamma-ray line signatures by Cherenkov telescopes (H.E.S.S. [28] and MAGIC [25]) and Fermi-LAT [29]. Annihilation into two gamma rays is assumed. Figure adapted from [25].

\subsection{Results from galaxy clusters}

MAGIC observed Perseus cluster for 24 hours in 2008, finding no gamma-ray signal [30]. The $\mathrm{J}$-factor for this target is $10^{17} \mathrm{GeV}^{2} \mathrm{~cm}^{-5}$, lower than other clusters of galaxies (the main purpose of Perseus observations was the attempt of detecting gamma rays from cosmic ray interactions in the intra-cluster medium).

Later, H.E.S.S. published their results based on observations of Fornax cluster for 15 hours in 2005. Fornax has a J-factor on the range $0.1-1 \times 10^{18} \mathrm{GeV}^{2} \mathrm{~cm}^{-5}$ and is one of the best candidates for dark matter searches in clusters. H.E.S.S. observations have resulted on limits to $\langle\sigma v\rangle$ in the $10^{-20} \mathrm{~cm}^{3} \mathrm{~s}^{-1}$ range [31]. These limits very much depend on the uncertainty in the dark matter profile, which can be of order 10-100 between different parameterizations and when considering the effect of substructures.

VERITAS observed Coma cluster (with a J-factor of $0.8 \times 10^{17} \mathrm{GeV}^{2} \mathrm{~cm}^{-5}$ ) for 19 hours during 2008 and obtained limits to $\langle\sigma v\rangle$ of $\sim 10^{-20} \mathrm{~cm}^{3} \mathrm{~s}^{-1}$ for dark matter particles of mass $1 \mathrm{TeV}$ annihilating into $b \bar{b}$ quarks [32].

\subsection{Satellite galaxies}

Satellite galaxies can be regarded as the smallest dark matter clumps that have started stellar activity. There are 25 known satellite galaxies at present, laying at distances up to $250 \mathrm{kpc}$. There is a subpopulation known as "ultra-faint", discovered in the Sloan Digital Sky Survey, with massto-light ratios as high as several thousand $M_{\odot} / L_{\odot}$ [20], which make them the most dark matter dominated objects known. This fact, together with the absence of other conventional gamma-ray sources and their relatively high and well-determined J-factors, make satellite galaxies one of the best astrophysical probes into the nature of dark matter. 
Satellite galaxies have been extensively observed with Cherenkov telescopes, accumulating a total of 280 hours on 9 different targets.

H.E.S.S. has observed Sagittarius dwarf [33] for 11 hours in 2006, Canis Major dwarf [34] for 10 hours in 2006 and Sculptor and Carina [35] between 2008 and 2009 for 12 and 15 hours, respectively. No gamma-ray signal has been found in any of them. The large uncertainties on Sagittarius and Canis Major prevent us from using this result to set limits on the dark matter particle properties. Using Carina observations $\left(J=4 \times 10^{17} \mathrm{GeV}^{2} \mathrm{~cm}^{-5}\right)$ they set the limit $\langle\sigma v\rangle<3 \times 10^{-22}$ $\mathrm{cm}^{3} \mathrm{~s}^{-1}$ for a dark matter particle of $3 \mathrm{TeV}$. Using observations of Sculptor $\left(J=4 \times 10^{17} \mathrm{GeV}^{2}\right.$ $\mathrm{cm}^{-5}$ ), the limit goes down to $\langle\sigma v\rangle=10^{-22} \mathrm{~cm}^{3} \mathrm{~s}^{-1}$ for a mass of $2 \mathrm{TeV}$.

VERITAS has observed Draco $\left(J=1.5 \times 10^{18} \mathrm{GeV}^{2} \mathrm{~cm}^{-5}\right)$ for 18 hours, Ursa Minor $(J=$ $\left.2.7 \times 10^{18} \mathrm{GeV}^{2} \mathrm{~cm}^{-5}\right)$ for 19 hours, Willman $1\left(\mathrm{~J}=8 \times 10^{18} \mathrm{GeV}^{2} \mathrm{~cm}^{-5}\right)$ for 14 hours, Boötes $1\left(J=1.2 \times 10^{18} \mathrm{GeV}^{2} \mathrm{~cm}^{-5}\right)$ for 14 hours [36] and Segue $1\left(J=1.1 \times 10^{19} \mathrm{GeV}^{2} \mathrm{~cm}^{-5}\right)$ for 48 hours [26], during the period between 2007 and 2011. The best limits are obtained from Segue 1 observations, with a limit $\langle\sigma v\rangle \sim 10^{-23} \mathrm{~cm}^{3} \mathrm{~s}^{-1}$ for a dark matter particle mass of $1 \mathrm{TeV}$, and assuming annihilation into $b \bar{b}$ quark pairs.

Finally, MAGIC has observed Draco for 8 hours [37], Willman 1 for 16 hours [38], and Segue 1 [39] for 29 hours between 2007 and 2009, all in single telescope mode. In addition, using the system of two telescopes, MAGIC has performed the deepest observations of a dwarf galaxy by Cherenkov telescopes so far, namely: 158 hours of Segue 1 between 2010 and 2013 [25]. The data from the observations were analyzed using, for the first time in Cherenkov astronomy, a full likelihood dedicated analysis [10], optimized for the recognition of spectral features, like the ones expected from dark matter annihilation or decay. The limits obtained go down to $\langle\sigma v\rangle \sim 5 \times$ $10^{-24} \mathrm{~cm}^{3} \mathrm{~s}^{-1}$ for dark matter particles of mass $1 \mathrm{TeV}$ annihilating into $b \bar{b}$ quark pairs. These are the strongest limits obtained from satellite galaxy observations by Cherenkov telescopes, and are stronger than the results by Fermi [27] for masses above $\sim 3 \mathrm{TeV}$ for annihilation into $b \bar{b}$, and above $\sim 600 \mathrm{GeV}$ for annihilation into $\tau^{+} \tau^{-}$.

or masses above few $\mathrm{TeV}$ for hadronic annihilation channels, and a few hundred $\mathrm{GeV}$ for leptonic channels (see Figure 1).

\subsection{The electron/positron spectrum}

Dark matter can modify the local spectrum of electron and positrons with respect to the expectations from pure secondary production by cosmic ray interactions with the interstellar medium. If dark matter is composed of Majorana particles, they may annihilate in the local Galactic halo, resulting in a symmetric production of particles and antiparticles, which may alter the overall positron content in the cosmic ray flux. In 2009, the PAMELA satellite detector measured a positron/electron fraction increasing in the energy range between 10 and $100 \mathrm{GeV}$, in a way that appears to be completely inconsistent with secondary sources [40] . This result was later confirmed and extended to higher energies by the AMS-02 detector [41] onboard the International Space Station. However, the results by PAMELA and AMS are insufficient to distinguish between astrophysical primary sources and dark matter annihilation, and measurements at higher energies are needed.

H.E.S.S. and MAGIC have measured the cosmic electron-positron spectrum between $100 \mathrm{GeV}$ and $5 \mathrm{TeV}[42,43]$. Electron and gamma-ray signals are in first approximation indistinguishable 


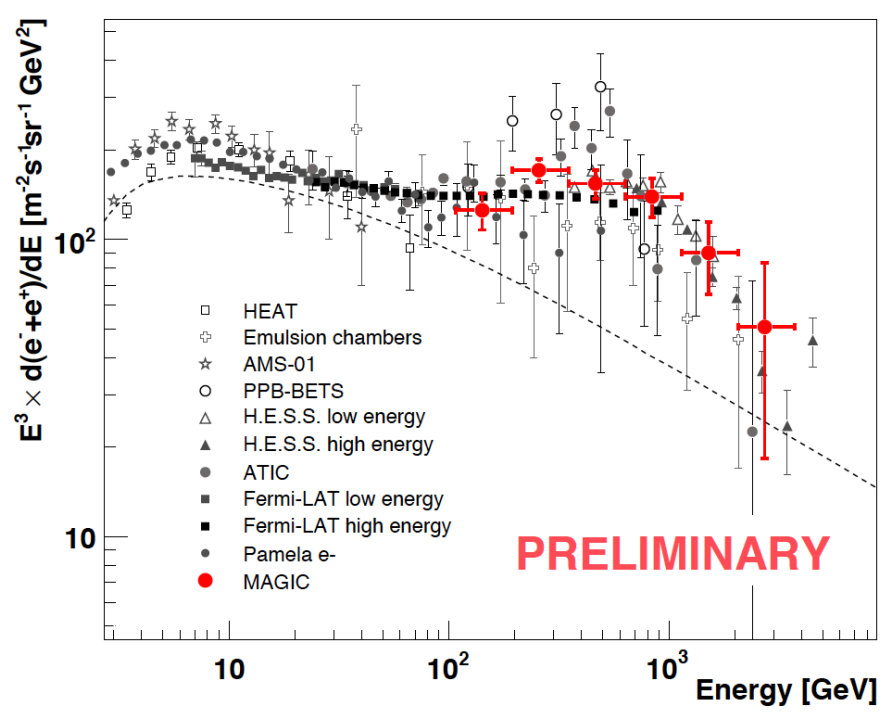

Figure 3: Electron-positron spectrum in the energy range between $3 \mathrm{GeV}$ and $5 \mathrm{TeV}$ measured by different experiments. Figure taken from [43].

for IACTs. The measurement is based on the fact that the former are the dominant electromagnetic component of the diffuse CR flux. The main background comes from hadronic CRs, and can be reduced using multi-variate statistical analyses that exploit the differences in the Cherenkov shower images between electrons and hadrons [45], which requires extensive MC simulations of shower development in the atmosphere. The results (see Figure 3) are in agreement, within systematic uncertainties, with what was observed by Fermi-LAT: the spectral feature claimed by [46] is not confirmed, and the spectrum is well fitted by a power law with index -3 , and a steepening starting at $\sim 1 \mathrm{TeV}$.

MAGIC is currently developing a novel method in Cherenkov astronomy to measure the fraction of positrons and electrons in the cosmic flux, in the energy range between $300 \mathrm{GeV}$ and 1 $\mathrm{TeV}$ [44]. This is possible by pointing the MAGIC telescopes towards the vicinity of the Moon. The Earth/Moon system forms a natural spectrometer in which the Moon absorbs a part of the cosmic rays creating a localized deficit in the isotropic flux (the so-called "Moon shadow") and the Earth magnetosphere deflects the trajectory of any incoming particle depending on its charge and momentum. Thanks the good angular and energy resolutions of MAGIC, the intensity of the positron and electron shadows can be measured with high accuracy -the ratio of these intensities is the cosmic positron/electron fraction. With this strategy, the measurement of the positron fraction can be performed up to $\sim 1 \mathrm{TeV}$. This is a very challenging measurement due to the high level of noise induced by the scattered moonlight during the observations. Although the major technical drawbacks of the technique have now been overcome, the detection of the electron Moon shadow with MAGIC will still require several years because of the short observation window available every year. It is possible to expand such window by using UV filters, which allow to observe under even higher camera illumination conditions. 


\section{Discussion and Conclusions}

Cherenkov telescopes have dedicated a (relatively small although significant) fraction of their time to search for dark matter signals, using different strategies and observational targets. Those include the galactic center region, dwarf galaxies, galaxy clusters, and the electron/positron diffuse flux. The most constraining results come from H.E.S.S. observations of the Galactic center halo, with limits on the velocity-averaged annihilation cross section down to $\langle\sigma v\rangle=3 \times 10^{-25} \mathrm{~cm}^{3} \mathrm{~s}^{-1}$ for hadronic channels. From dwarf galaxies, MAGIC produces the most constraining results using very deep observations of the Segue 1 galaxy, with limits down to $\langle\sigma v\rangle \sim 5 \times 10^{-24} \mathrm{~cm}^{3} \mathrm{~s}^{-1}$. The bounds obtained with Cherenkov telescopes are more constraining that those obtained with Fermi-LAT above few hundred GeV.

The sensitivity of this kind of searches is determined by four factors:

- The sensitivity of the instrument(s)

- The optimization of the analysis

- The depth of the observations

- The choice of optimal targets

The analysis described in [10] and successfully applied by MAGIC in [25] seems to be the way of fully exploiting the characteristic spectral features in dark matter-induced processes (it yields a factor of $\sim 2$ better sensitivity than the conventional analysis for this kind of searches). In addition, the current instruments have already invested a significant amount of time and little gain can be expected by adding few tens of hours by each of the telescopes individually. In this sense, one could conclude that Cherenkov telescopes have already produced their result, and no news should be expected before the advent of CTA [47]. However, new targets can be discovered (or the properties of existing ones better determined), particularly new satellite galaxies. The use of a likelihood analysis allows the combination of results from different targets and by different instruments in a relatively trivial way [10]. A natural way to obtain a global, sensitivity optimized result on dark matter by the current generation of Cherenkov instruments should be the combination of present and future results.

\section{References}

[1] P. A. R. Ade et al., Planck 2013 results. XVI. Cosmological parameters, Astron. and Astrophys. 571 (2014) A16. [arXiv:1303.5076].

[2] P. Hut, Limits on masses and number of neutral weakly interacting particles, Phys. Lett. B69 (1977) 85.

[3] O. Buchmueller, Searches for Supersymmetry, PoS (EPS-HEP 2013) 161 (2013).

[4] F. Arneodo, Dark Matter Searches, in Proc. of XXXII Symposium on "Physics in Collision", Strbske Pleso, Slovakia, 2013. [arXiv:1301.0441].

[5] M. Cahill-Rowley et al., Complementarity and Searches for Dark Matter in the pMSSM, arXiv:1305.6921 (2013). 
[6] G. Bertone (ed.), Particle Dark Matter. Cambridge University Press. Cambridge (2010).

[7] D. Hooper and P. D. Serpico, Detecting Axionlike Particles with Gamma Ray Telescopes, Phys. Rev. Lett. 99 (2007) 231102. [arXiv: 0706 .3203].

[8] A. De Angelis, M. Roncadelli and O. Mansutti, Evidence for a new light spin-zero boson from cosmological gamma-ray propagation? Phys. Rev. D 76 (2007), 121301. [arXiv: 0707.4312 ].

[9] M. Doro et al., Dark matter and fundamental physics with the Cherenkov Telescope, Astropart. Phys. 43(2013) 189. [arXiv:1208.5356].

[10] J. Aleksić, J. Rico and M. Martinez, Optimized analysis method for indirect dark matter searches with Imaging Air Cherenkov Telescopes, JCAP 10 (2012) 032. [arXiv: 1209.5589 ].

[11] T. Bringmann et al., New gamma-ray contributions to supersymmetric dark matter annihilation, JHEP 01 (2008) 049. [arXiv: 0710 . 3169].

[12] J. A. R. Cembranos et al., Photon spectra from WIMP annihilation, Phys. Rev. D83 (2011) 083507. [arXiv:1009.4936].

[13] D. Merritt et al., Empirical Models for Dark Matter Halos. I. Nonparametric Construction of Density Profiles and Comparison with Parametric Models, Astron. J. 132 (2006) 2685. [astro-ph/0509417].

[14] J. F. Navarro, C. S. Frenk and S. D. M. White, The Structure of Cold Dark Matter Halos, Astrophys. J. 462 (1996) 563. [astro-ph/9508025].

[15] M. Rocha et al., Cosmological simulations with self-interacting dark matter - I. Constant-density cores and substructure, MNRAS 430 (2012) 81. [arXiv: 1208 . 3025].

[16] J. Zavala et al., Constraining self-interacting dark matter with the Milky Way's dwarf spheroidals, MNRAS 431 (2013) L20. [arXiv:1211. 6426].

[17] K. K. Boddy et al., Self-interacting dark matter from a non-Abelian hidden sector, Phys. Rev. D89 (2014) 115017. [arXiv:1402.3629].

[18] J. Aleksić et al., MAGIC Gamma-ray Telescope Observation of the Perseus Cluster of Galaxies: Implications for Cosmic Rays, Dark Matter, and NGC 1275, Astrophys. J 710 (2010) 634. [arXiv:0909.3267].

[19] A. Abramowski et al., Search for a Dark Matter annihilation signal from the Galactic Center halo with H.E.S.S. . Phys. Rev. Lett. 106 (2011) 161301. [arXiv: 1103.3266 ].

[20] L. E. Strigari, Galactic searches for dark matter, Phys. Rep. 531 (2013) 1. [arXiv: 1211 . 7090 ].

[21] J. Rico, Gamma ray astronomy: implications for fundamental physics, in Proc. of XXXI Inter. Symp. "Physics in Collision", Vancouver, Canada (2011).

[22] F. Aharonian et al., H.E.S.S. observations of the Galactic Center region and their possible dark matter interpretation, Phys. Rev. Lett. 97 (2006) 221102. [astro-ph/ 061050 9].

[23] J. Albert et al., Observation of Gamma Rays from the Galactic Center with the MAGIC telescope, Astrophys. J. 638 (2006) L101. [astro-ph/ 0512469 ].

[24] A. Archer et al., Very-High Energy Observations of the Galactic Center Region by VERITAS in 2010-2012, Astrophys. J. 790 (2014) 149. [arXiv: 1406.6383 ].

[25] J. Aleksić et al., Optimized dark matter searches in deep observations of Segue 1 with MAGIC, JCAP 02 (2014) 008. [arXiv: 1312 .1535]. 
[26] E. Aliu et al., VERITAS Deep Observations of the Dwarf Spheroidal Galaxy Segue 1. Phys. Rev. D85 (2012) 062001. [arXiv:1202.2144].

[27] M. Ackermann et al., Dark Matter Constraints from Observations of 25 Milky Way Satellite Galaxies with the Fermi Large Area Telescope, Phys. Rev. D89 (2014) 042001. [arXiv: 1310 . 0828].

[28] A. Abramowski et al., Search for photon line-like signatures from Dark Matter annihilations with H.E.S.S, Phys. Rev. Lett. 110 (2013) 041301. [arXiv: 1301.1173 ].

[29] M. Ackermann et al., Search for gamma-ray spectral lines with the Fermi Large Area Telescope and dark matter implications, Phys. Rev. D88 (2013) 082002. [arXiv: 1305.5597 ].

[30] J. Aleksić et al., MAGIC Gamma-Ray Observation of the Perseus Galaxy Cluster, Astrophys. J. 710 (2010) 634. [arXiv:0909.3267].

[31] A. Abramowski et al., Search for Dark Matter Annihilation Signals from the Fornax Galaxy Cluster with H.E.S.S , Astrophys. J. 750 (2012) 123 [arXiv: 1202.5494 ]. Erratum: ibid 783 (2014) 63.

[32] T. Arlen et al., Constraints on Cosmic Rays, Magnetic Fields, and Dark Matter from Gamma-Ray Observations of the Coma Cluster of Galaxies with VERITAS and Fermi, Astrophys. J. 757 (2012) 123. [arXiv:1208.0676].

[33] F. Aharonian et al., Observations of the Sagittarius Dwarf galaxy by the H.E.S.S. experiment and search for a Dark Matter signal, Astropart. Phys. 29 (2008) 55. [arXiv: 0711.2369 ]. Erratum: ibid 33 (2010) 274.

[34] F. Aharonian et al., A search for a dark matter annihilation signal towards the Canis Major overdensity with H.E.S.S, Astrophy. J. 691 (2009) 175. [arXiv: 0809.3894 ].

[35] A. Abramowski et al., H.E.S.S. constraints on Dark Matter annihilations towards the Sculptor and Carina Dwarf Galaxies, Astropart. Phys. 34 (2011) 608. [arXiv: 1012.5602 ].

[36] V. A. Acciari et al., VERITAS Search for VHE Gamma-ray Emission from Dwarf Spheroidal Galaxies. Astrophys. J. 720 (2010) 1174. [arXiv: 1006.5955$].$

[37] J. Albert et al., Upper limit for gamma-ray emission above $140 \mathrm{GeV}$ from the dwarf spheroidal galaxy Draco. Astrophys. J. 679 (2008) 428. [arXiv: 0711.2574 ].

[38] J. Albert et al., Upper Limits on the VHE Gamma-Ray Emission from the Willman 1 Satellite Galaxy with the Magic Telescope. Astrophys. J. 697 (2009) 1299. [arXiv: 0810.3561$].$

[39] J. Aleksić et al., Searches for dark matter annihilation signatures in the Segue 1 satellite galaxy with the MAGIC-I telescope, JCAP 06 (2011) 035. [arXiv: 1103.0477 ].

[40] O. Adriani et al., An anomalous positron abundance in cosmic rays with energies 1.5-100 GeV, Nature 458 (2009) 607. [arXiv: 0810 . 4995].

[41] M. Aguilar et al., First Result from the Alpha Magnetic Spectrometer on the International Space Station: Precision Measurement of the Positron Fraction in Primary Cosmic Rays of 0.5-350 GeV, Phys. Rev. Lett. 110 (2013) 141102.

[42] F. Aharonian et al., Probing the ATIC peak in the cosmic-ray electron spectrum with H.E.S.S, Astron. and Astrophys. 508 (2009) 561. [arXiv:0905.0105].

[43] D. Borla-Tridon et al., Measurement of the cosmic electron plus positron spectrum with the MAGIC telescopes. In Proc. of the 32nd International Cosmic Ray Conference, [arxiv:1110.4008]. 
[44] P. Colin et al., Probing the CR positron/electron ratio at few hundreds GeV through Moon shadow observation with the MAGIC telescopes. In Proc. of the 32nd International Cosmic Ray Conference, [arxiv: 1110.0183$]$.

[45] J. Albert et al., Implementation of the Random Forest Method for the Imaging Atmospheric Cherenkov Telescope MAGIC, Nucl. Instr. and Meth. A588 (2008) 424. [arXiv: 0709.3719$].$

[46] J. Chang et al., An excess of cosmic ray electrons at energies of 300-800 GeV, Nature 456 (2008) 362.

[47] B. S. Acharya et al., Introducing the CTA concept, Astropart. Phys. 43 (2013) 3 